\title{
Metallopanstimulin-1 (MPS-1) mediates the promotion effect of leptin on colorectal cancer through activation of JNK/c-Jun signaling pathway
}

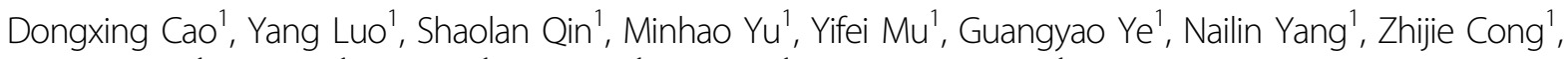
Jianjun Chen ${ }^{1}$, Jun Qin ${ }^{1}$, Ran Cui ${ }^{1}$, Ran Jing ${ }^{1}$, Hui Cao ${ }^{1}$ and Ming Zhong ${ }^{1}$

\begin{abstract}
Obesity is a major epigenetic cause for colorectal cancer (CRC). Leptin is implicated in obesity-associated CRC, but the underlying mechanism remains unclear. The current study identified over-expression of metallopanstimulin-1 (MPS-1) in CRC patients through microarray and histological analysis, especially in obese CRC patients. MPS-1 was correlated with advanced tumor stage, suggesting its association with CRC progression. In addition, MPS-1 over-expression was associated with poor overall survival (OS) in obese CRC patients, but not in their non-obese counterparts, suggesting its potential as a prognostic marker of obese CRC patients. MPS-1 expression was positively associated with circulating leptin levels in CRC patients, especially in obese cases. Functional experiments demonstrated that MPS-1 silencing inhibited tumor proliferation and colony formation, and induced apoptosis of CRC cells in vitro. Converse results were obtained from the experiments with MPS-1 over-expression. Mechanistically, MPS- 1 executed its action through induction of c-Jun N-terminal kinase (JNK)/C-Jun pathway. Moreover, the promotion effect of MPS-1 on CRC progression was modulated by leptin. In vivo studies demonstrated that MPS-1 silencing suppressed tumor growth of CRC via inhibiting JNK/C-Jun signaling. Collectively, this study indicates that MPS-1 promotes leptin-induced CRC via activating JNK/c-Jun pathway. MPS-1 might represent a potent candidate for the treatment and prognostic prediction of obesity-associated CRC.
\end{abstract}

\section{Introduction}

Obesity is a global health challenge with a number of co-morbidities including enhanced risk of colorectal cancer $(\mathrm{CRC})^{1}$. Among proposed mechanisms linking adiposity to CRC, much attention has been drawn to the deregulation of adipokine signaling, with a focus on lep$\mathrm{tin}^{2}$. Leptin is an adipocyte-derived hormone that is upregulated in obesity and plays a central role in regulating energy homeostasis ${ }^{3}$. Despite emerging evidences support leptin as a mediator of obesity-associated $\mathrm{CRC}^{4}$, the mechanisms underlying this pathobiology remain unclear.

Correspondence: Ming Zhong (surgeonzhongming@163.com)

'Department of Gastrointestinal Surgery, Ren Ji Hospital, School of Medicine, Shanghai Jiao Tong University, 200127 Shanghai, China

Edited by A. Stephanou
Metallopanstimulin-1 (MPS-1), also known as ribosomal protein S27 (RPS27), is a multifunctional protein ubiquitously expressed in most normal human tissues ${ }^{5}$. MPS-1 plays a multifaceted role in different types of tumors. While MPS-1 was regarded as a tumor suppresser in head and neck squamous cell carcinoma ${ }^{6}$, it was found up-regulated in several forms of malignancies, such as breast cancer $^{7,8}$ and gastric cancer ${ }^{9-11}$. For example, previous studies have revealed that knockdown of MPS-1 could inhibit growth and induce apoptosis of gastric cancer cells both in vitro and in vivo by suppressing NF$\kappa B$-signaling pathway ${ }^{11}$. So far, the implication of MPS-1 in CRC has rarely been reported except in a case report. In a patient with CRC, Ganger et al. observed that MPS-1 over-expression in tumor tissues and negative expression in the adjacent normal mucosa, and proposed a

\section{(c) The Author(s) 2019}

(c) (i) Open Access This article is licensed under a Creative Commons Attribution 4.0 International License, which permits use, sharing, adaptation, distribution and reproduction c. in any medium or format, as long as you give appropriate credit to the original author(s) and the source, provide a link to the Creative Commons license, and indicate if changes were made. The images or other third party material in this article are included in the article's Creative Commons license, unless indicated otherwise in a credit line to the material. If material is not included in the article's Creative Commons license and your intended use is not permitted by statutory regulation or exceeds the permitted use, you will need to obtain permission directly from the copyright holder. To view a copy of this license, visit http://creativecommons.org/licenses/by/4.0/. 
correlation between MPS-1 with a more aggressive behavior of $\mathrm{CRC}^{12}$. Nevertheless, results from large cohort studies and functional experiments are lacking.

Here, using microarray and histological analysis, we identified MPS-1 over-expression in CRC, especially in obese CRC patients. We evaluated the association between MPS-1 and prognosis and circulating leptin level of CRC patients, especially in the context of obesity. Functionally, the influences of MPS-1 on CRC progression and the underlying mechanism were investigated in vitro and in vivo. The crosstalk between MPS-1 and leptin was also assessed. These findings outline a role for MPS-1 in leptin-related CRC.

\section{Materials and methods}

\section{Patients recruitment}

The study included three independent patient cohorts at Department of Gastrointestinal Surgery, Ren Ji Hospital, School of Medicine, Shanghai Jiao Tong University. Cohort 1 included five CRC patients who received colectomy during January 2016-June 2018 for microarray analysis. Cohort 2 consisted of 40 CRC patients (20 obese and 20 non-obese cases) who received colectomy during January 2016-June 2018. Cohort 2 was included to verify the findings in microarray and to investigate the correlation between MPS- 1 and serum leptin. Cohort 3 recruited 155 patients (83 obese and 72 non-obese cases) who underwent colectomy during January 2012-June 2014 for survival analysis. The patients who did not possess integrated follow-up information were excluded from cohort 3. All patients were confirmed CRC by pathological diagnosis. Tumor staging was performed according to American Joint Committee on Cancer (AJCC) 8th edition manual. Obesity was defined by body mass index $(\mathrm{BMI}) \geq$ $30 \mathrm{~kg} / \mathrm{m}^{2}$ according to the World Health Organization (WHO) classification ${ }^{13}$. Tumors and normal colon tissues were collected during operation. Normal colon tissues meant adjacent colonic mucosa more than $10 \mathrm{~cm}$ away from the tumor. The procedures were approved by the hospital Clinical Research Ethics Committee and informed consents were obtained.

\section{Microarray analysis}

Total RNA was extracted from tissues by Trizol reagent (Invitrogen, Carlsbad, CA, USA). RNA quantity and quality were assessed with Thermo Nanodrop 2000 (Thermo Fisher Scientific, Wilmington, DE, USA) and Agilent 2100 Bioanalyzer (Agilent, Santa Clara, CA, USA). Affymetrix PrimeView Human Gene Expression Arrays (Thermo Fisher Scientific, Willmington, DE, USA) were applied for microarray analysis according to the manufacturer's instruction. RNA was labeled by biotin using GeneChip 3' IVT Express kit (Affymetrix). After hybridization overnight, the chips were stained by GeneChip ${ }^{\text {tx }}$
Hybridization wash and stain kit (Affymetrix), and scanned with GeneChip Scanner 3000 according to the manufacturer's protocol. Significantly differentially expressed genes were selected based on this threshold: FDR $<0.05$ and absolute fold change $>2$.

\section{Cell culture}

Human CRC cell lines RKO, DLD-1, HT29, SW480, HCT116, and Caco2, and normal colon epithelial cell FHC were purchased from BeNa Technology (Beijing, China). Cells were grown in six-well plates at $37^{\circ} \mathrm{C}$ in a humidified atmosphere containing $5 \% \mathrm{CO}_{2}$ and $95 \%$ air. SW480 cells were cultured using DMEM (Invitrogen, Carlsbad, CA, USA) supplemented with 10\% FBS (Gibco, Rockville, MD, USA). RKO, HCT116, DLD-1, and FHC cells were cultured using RPMI1640 (Thermo Fisher Scientific, Wilmington, DE, USA) with 10\% FBS. HT-29 was cultured with McCoy's 5A medium (Thermo Fisher Scientific) with $10 \% \mathrm{FBS}$. $\mathrm{CaCo}_{2}$ was cultured with MEM (Thermo Fisher Scientific) supplemented with 20\% FBS. Cell culture medium was changed every $72 \mathrm{~h}$. To detect the impact of leptin, cells were cultured in the presence or absence of recombinant human leptin $(50 \mathrm{ng} / \mathrm{mL}$, \# L4146, Sigma, Merck, Darmstadt, Germany) for $24 \mathrm{~h}$. To detect the impact of anisomycin, cells were cultured in the presence or absence of anisomycin $(0.01 \mu \mathrm{M}$, Beyotime, Shanghai, China) for $48 \mathrm{~h}$.

\section{Real-time qPCR}

Total RNA was extracted using TRIzol $^{\oplus}$ reagent (Thermo Fisher Scientific, Wilmington, DE, USA) according to the manufacturer's protocol. The purity and integrity of RNA was assessed by Nanodrop 2000 spectrophotometry. Then, $\mathrm{cDNA}$ was generated by HiScript $\mathrm{Q}$ RT SuperMix for qPCR (+gDNA wiper) (Vazyme, Nanjing, Jiangsu, China) according to the manufacturer's protocol. qPCR was performed using AceQ qPCR SYBR Green Master Mix (Vazyme). Gene expression was calculated using the comparative $\mathrm{Ct}$ method. GAPDH was used as the endogenous control. The primers for qPCR are listed in Table S1.

\section{Western blotting (WB)}

CRC tissues and cells and FHC cells were lysed in icecold radio immunoprecipitation assay (RIPA) buffer (Millipore, Temecula, CA, USA) for $30 \mathrm{~min}$. Total protein concentration was determined with BCA Protein Assay Kit (Thermo Fisher Scientific, Waltham, MA, USA). Protein samples $(20 \mu \mathrm{g}$ in each lane) were separated by 10\% SDS-PAGE (Invitrogen, Carlsbad, CA, USA), and were subsequently transferred onto polyvinylidene fluoride (PVDF) membrane. Blots were incubated with 5\% BSA in Tris-buffered saline containing $0.5 \%$ Tween 20 (TBST) for $60 \mathrm{~min}$, and incubated overnight at $4{ }^{\circ} \mathrm{C}$ with 
corresponding primary antibodies. Membranes were then incubated for $1 \mathrm{~h}$ in appropriate secondary antibody. Proteins were visualized by enhanced chemiluminescence (ECL). Antibodies are listed in Table S2.

\section{Immunohistochemical staining (IHC)}

Paraffin-embedded CRC and normal tissue samples from CRC patients were cut into $4 \mu \mathrm{m}$ sections. The samples were blocked and then incubated with anti-MPS-1, anti-pJNK (c-Jun N-terminal kinase), anti-p-c-Jun, and anti-Ki-67 at $4{ }^{\circ} \mathrm{C}$ overnight in incubator. Next, the sections were incubated with goat anti-rabbit IgG H\&L horseradish peroxidase (HRP)-conjugated secondary antibody at $37^{\circ} \mathrm{C}$ for $1 \mathrm{~h}$. Finally, tissue sections were stained with $3,3^{\prime}$-diaminobenzidine (DAB) and subsequently hematoxylin at room temperature. Images were captured using a photomicroscope (Olympus, Shinjuku, Tokyo, Japan) and analyzed. The staining intensity was scored as 0 (negative), 1 (weak), or 2 (strong). The staining extent was graded as $0(0 \%), 1$ (1-25\%), 2 (26-50\%), 3 (51-75\%), or 4 (76-100\%). The samples were classified into high and low expression groups based on the sum of staining intensity and extent. Antibodies are listed in Table S2.

\section{Detection of serum leptin level}

Blood samples were drawn from cohort 2 before operation. The samples were separated through centrifugation at $800 \times g$ for $30 \mathrm{~min}$. The serum concentrations of leptin were determined by Leptin Human ELISA Kit (\# KAC2281, Thermo Fisher Scientific, Waltham, MA, USA) according to the manufacturer's protocol.

\section{Vectors construction and lentivirus transfection}

For over-expression, the MPS- 1 construct was generated by subcloning human MPS-1 cDNA into vector BRV-214 (Shanghai Biosciences, Shanghai, China). Lentivirus production and transfection were performed as previously described $^{14}$. For gene knockdown, target short hairpin RNA (shRNA) sequence or scramble shRNA (shCtrl) was packaged into vector BR-V-215 (Shanghai Biosciences) using Fermentas T4 DNA Ligase (New England Biolabs). Plasmids containing target shRNA sequences were extracted with EndoFree Plasmid Maxi Kit (Qiagen, Valencia, CA, USA). Cell transfection was conducted with Lipofectamine 2000 (Thermo Fisher Scientific). The cells were screened under Puromycin (Takara Bio, Otsu, Japan) and verified by detecting GFP fluorescence with a fluorescence microscope (Olympus, Tokyo, Japan). The target sequences and corresponding shRNA sequences are listed in Table S3.

\section{CCK8 assay}

Cell proliferation was measured with the Cell Counting Kit-8 (Dojindo, Kumamoto, Japan) according the instruction of manufacturer. Briefly, RKO, HCT116, and Caco2 cells were seeded in 96-well plates with $100 \mu \mathrm{L}$ medium (3000 cells/well). The measurement was performed at 1, 2, 3, 4, and 5 days after seeding. After CCK-8 reagent was added $(10 \mu \mathrm{L} /$ well $)$, the cells were cultured at $37^{\circ} \mathrm{C}$ until visual color conversion occurred. The absorbance was determined at $450 \mathrm{~nm}$ with a microplate reader (Tecan, Männedorf, Zürich, Switzerland). Inhibition rate was calculated by the equation: [OD (shCtrl)-OD (shGene)]/OD (shCtrl).

\section{Colony formation assay}

Four days after cell transfection, RKO, HCT116, and Caco2 cells in logarithmic growth phase were digested by trypsin, and seeded in six-well plates (500 cells/well). Cells were cultured in the medium at $37^{\circ} \mathrm{C}$ for 14 days to form colonies. Then cells were washed by PBS, fixed by $4 \%$ paraformaldehyde for $1 \mathrm{~h}$, stained with Giemsa $(500 \mu \mathrm{L})$ for $20 \mathrm{~min}$, and photographed with a digital camera. The number of colonies ( $>50$ cells/colony) was counted under fluorescence microscopy (MicroPublisher 3.3 RTV, Olympus, Tokyo, Japan).

\section{Cell apoptosis flow cytometry assay}

The quantitative assessment of apoptosis was performed with flow cytometry. Briefly, RKO, HCT116, and Caco2 cells were plated on six-well plates at a density of $2 \times 10^{5}$ cells/well to assess apoptosis. After incubation for $24 \mathrm{~h}$, cells were harvested and pelleted by centrifugation for Annexin $\mathrm{V}$ staining with a eBioscience Annexin V Apoptosis Detection Kit APC (Invitrogen, Carlsbad, CA, USA). Cell pellets were washed twice with phosphate buffer and resuspended in $250 \mu \mathrm{L}$-binding buffer at a density of $1 \times 10^{6}$ cells $/ \mathrm{mL}$. The cells were stained with $5 \mu \mathrm{L}$ Annexin $\mathrm{V}$ and incubated on ice for $15 \mathrm{~min}$. The cell suspension $(100 \mu \mathrm{L})$ was analyzed by flow cytometry to detect apoptosis.

\section{Caspase $3 / 7$ activity}

Apoptotic cell death was quantified by using the Caspase-Glo ${ }^{\oplus}$ /7 assay kit (Promega, Madison, WI, USA) according to the manufacturer's protocol. Briefly, RKO, HCT116, and Caco 2 cells at 100,000 cells/well were seeded into 96-well plates. Before detection, $100 \mu \mathrm{L}$ of Caspase-Glo reagent was added to each well and incubated for $2 \mathrm{~h}$. The luminescence was measured with a microplate reader (Tecan Infinite, Groedig, Austria).

\section{Mice xenograft model}

BALB/c male nude mice (6 weeks of age) were purchased from Shanghai Jiesijie Experimental Animals Co., Ltd (Shanghai, China). Mice were randomly divided into shMPS-1 group and shCtrl group (6 mice/group). RKO cells $\left(5 \times 10^{6}\right.$ cells/mouse) transfected with shMPS- 1 or shCtrl were subcutaneously injected into mice. Tumor 
growth was measured in volume (calculated by the equation $\pi / 6 \times L \times W^{2}$, where $L$ represents longest dimension and $W$ means dimension perpendicular to length) on days $6,12,18,24$ post inoculation. Mice were sacrificed 25 days after injection and tumor weights were measured. All procedures were approved by the Institutional Animal Care and Use Committee of Shanghai Jiao Tong University.

\section{Statistical analysis}

Results were presented as mean $\pm \mathrm{SD}$. Chi-square test was used for categorical variables. Continuous variables were compared with Student's $t$-test (two groups) or One-way ANOVA followed by Bonferroni post-hoc test (three or more groups). Linear regression analysis was used to evaluate the relationship between MPS-1 and serum leptin level. Kaplan-Meier survival curves with log-rank test was used to analyze overall survival (OS). Each experiment was repeated three times. $P<0.05$ was defined as statistically significant (two-sided). Statistical analysis was performed using SPSS 14.0 for Windows (SPSS Inc., Chicago, IL, USA).

\section{Results}

MPS-1 expression was identified as a promoter in CRC, especially in obese CRC patients

To explore novel regulators in CRC, gene expression profiles in five pairs of tumor and normal tissues from cohort 1 were detected by microarray analysis. Transcriptome profiling identified 1140 up-regulated and 1091 down-regulated genes in CRC tissues (Fig. 1a, Table S4). Subsequently, the inhibition impacts of 10 up-regulated candidates on the proliferation of RKO cells were detected by gene knockdown experiments. The results indicated that MPS-1 displayed the largest inhibition rate on cell proliferation $(P<0.05$, Fig. 1b, Table S4).

To validate the findings in microarray screening, MPS-1 expression was determined in 40 pairs of tumor and normal tissues from cohort 2. Despite the up-regulation in all patients $(P<0.001$, Fig. $1 \mathrm{c}-\mathrm{e})$, MPS-1 mRNA levels showed a higher fold up-regulation in obese CRC patients than in their non-obese counterparts (1.40-fold in nonobese group, 2.12-fold in obese group, Fig. 1c, d). The results of WB and IHC demonstrated similar results, as shown by the representative images in Fig. 1f, g. Notably, the analysis of MPS-1 expression and basic characteristics of cohort 3 found an association between MPS-1 expression and advanced tumor stage $(P<0.001$, Table 1$)$. These findings suggested MPS- 1 as a promoter in the development and progression of CRC.

\section{MPS-1 expression was correlated with poor prognosis and circulating leptin level in obese CRC patients}

In order to determine the relationship between MPS-1 and prognosis of CRC, its impact on OS was preliminarily investigated through data mining of The Cancer Genome Atlas (TCGA). No correlation was found between MPS-1 expression and OS $(P>0.05$, Fig. 2a). Furthermore, we performed subgroup analysis in cohort 3 according to BMI. Kaplan-Meier analysis indicated that MPS-1 overexpression was correlated with poor OS in obese CRC patients $(P=0.038$, Fig. $2 \mathrm{~b})$ instead of non-obese cases $(P$ $>0.05$, Fig. $2 \mathrm{c})$, which might contribute to the statistical insignificance in survival analysis of all patients $(P>0.05$, Fig. 2d).

Moreover, the relationship between MPS-1 and serum leptin was analyzed to investigate its role in obese CRC patients. With a significant overall correlation $(P<0.001$, Fig. 2e), a positive correlation was found between MPS-1 expression and leptin level in obese CRC patients $\left(R^{2}=\right.$ $0.255, P=0.023$, Fig. 2f) and in all CRC patients from cohort $2\left(R^{2}=0.220, P=0.002\right.$, Fig. $\left.2 \mathrm{~g}\right)$ but not in nonobese cases $\left(R^{2}=0.025, P>0.05\right.$, Fig. $\left.2 \mathrm{~h}\right)$. These observations suggested that MPS-1 might be implicated in obesity-associated CRC through interacting with leptin.

\section{MPS-1-induced tumor growth and suppressed apoptosis in CRC cells}

Next, the impact of alteration in MPS-1 levels on CRC cells was investigated in vitro. As shown in Fig. S1a and $\mathrm{S} 1 \mathrm{~b}$, the expression of MPS-1 in CRC cells was upregulated compared with normal colon cell FHC $(P<$ 0.01 ), suggesting its role as a promoter in CRC. Subsequently, MPS-1 gene was knockdown in RKO cells and over-expressed in Caco 2 cells, respectively. The alteration in the expression of MPS-1 was verified at mRNA and protein levels $(P<0.01$, Fig. 3a, b, g, h, S2a, S2b). As shown in Figs. 3c, d and S2c, S2d, the knockdown of MPS1 suppressed cell proliferation $(25.6 \%$ inhibition rate for RKO cells, $P<0.01 ; 24.4 \%$ inhibition rate for HCT116 cells, $P<0.01)$ and colony formation $(65.4 \%$ inhibition rate for RKO cells, $P<0.01 ; 69.1 \%$ inhibition rate for HCT116 cells, $P<0.001$ ) compared with the negative control. Moreover, the down-regulation of MPS-1 promoted apoptosis in CRC cells (4.88-fold in RKO cells, $P<$ 0.001 ; 4.72 -fold in HCT116 cells, $P<0.001$ ) (Figs. 3e and S2e). Besides, enhanced activity of Caspase $3 / 7$ was detected (1.54-fold in RKO cells, $P<0.001 ; 2.04$-fold in HCT116 cells, $P<0.001)$, which may be an explanation for the enhanced apoptosis (Figs. 3f and S2f). Consistent with this, the over-expression of MPS-1 showed exactly conversed effect on CRC cells $(P<0.01$, Fig. 3i-l). Collectively, the results demonstrated that MPS-1 plays a promotion role in the development of CRC.

\section{Leptin alleviated the inhibition effect of MPS-1 knockdown on CRC cells}

Given that alterations in MPS-1 expression predominantly occurred in obese CRC patients, and MPS-1 


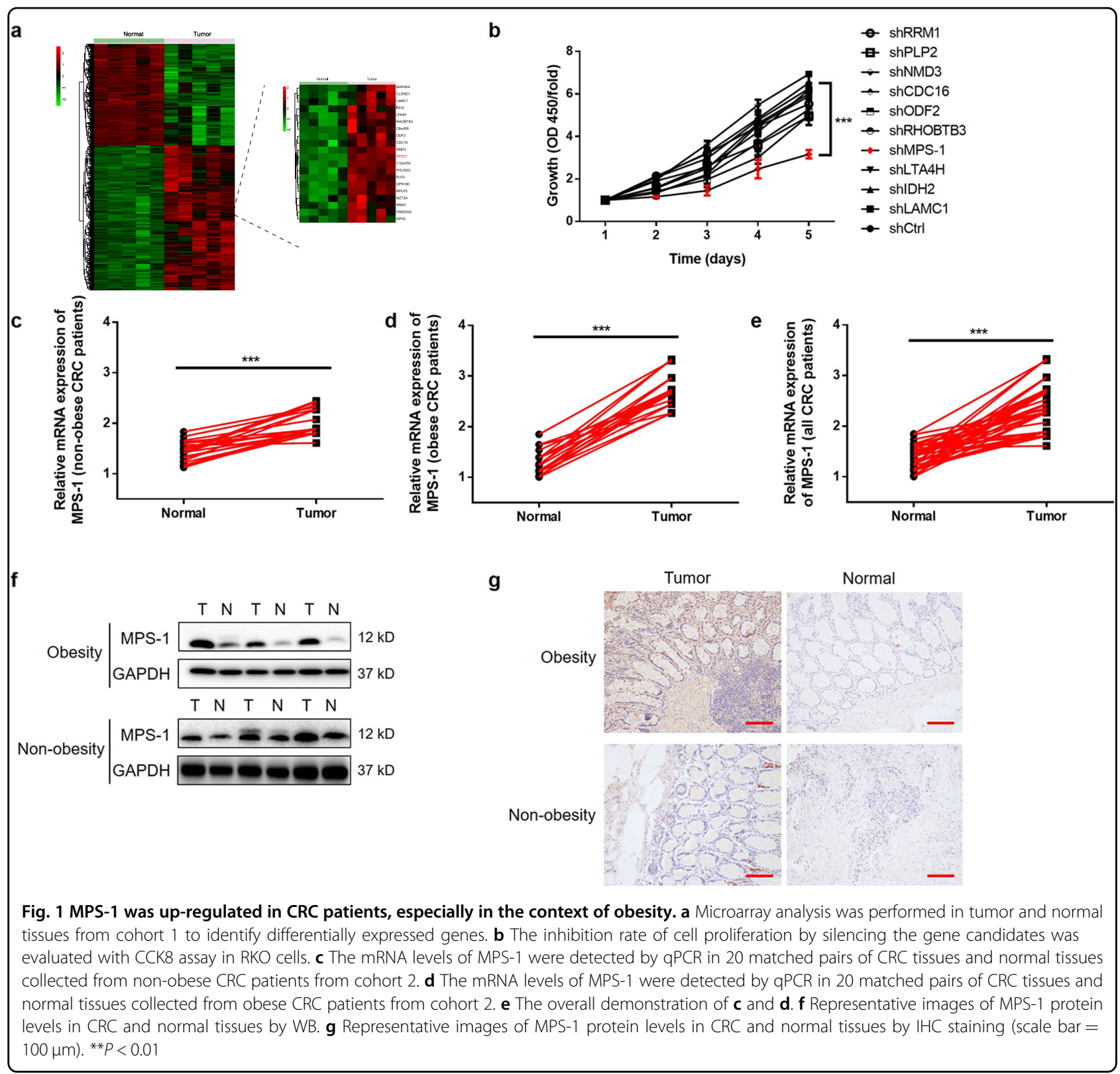

expression was correlated with serum leptin in obese CRC patients, we focused our attention on the relationship between MPS-1 and leptin. The impact of leptin on CRC cells with MPS-1 knockdown was evaluated with pharmacologic enhancement of leptin. The results of qPCR proved that treatment of leptin partially reverted the effect of MPS-1 gene knockdown (34.07\% recovery for RKO, $45.26 \%$ recovery for HCT116, $P<0.01$, Fig. $4 a)$. Subsequently, it was observed that leptin exposure partially reversed the effect of MPS-1 knockdown on cell proliferation (40.68\% recovery for RKO, 41.46\% recovery for HCT116) and colony formation (25.01\% recovery for RKO, 32.87\% recovery for HCT116) in both RKO and HCT116 cells $(P<0.01$, Fig. 4 b, c). Additionally, leptin exposure significantly alleviated the promotion effect of MPS-1 knockdown on cell apoptosis $(62.34 \%$ recovery for RKO, 66.76\% recovery for HCT116) and Caspase-3/7 activity (42.63\% recovery for RKO, $48.39 \%$ recovery for HCT116) in RKO and HCT116 cells $(P<0.05$, Fig. $4 \mathrm{~d}$, e). Taken together, the data demonstrated that MPS-1 modulated the development of CRC under the regulation of leptin.

\section{MPS-1 promoted CRC through activation of JNK/C-Jun- signaling pathway}

In order to investigate the mechanisms of the promotion effect of MPS-1 on CRC, protein levels of JNK/c-Jun pathway including JNK, p-JNK, c-Jun, and p-c-Jun (Ser- 
63/73) were measured by WB. As shown in Fig. 5a, the activities of JNK and c-Jun were suppressed as illustrated

Table 1 Relationship between MPS-1 expression and characteristics of patients of cohort 3

\begin{tabular}{|c|c|c|c|c|}
\hline \multirow[t]{2}{*}{ Features } & \multirow[t]{2}{*}{ No. of patients } & \multicolumn{2}{|c|}{ MPS-1 expression } & \multirow[t]{2}{*}{$P$-value } \\
\hline & & low & high & \\
\hline All patients & 155 & 87 & 68 & \\
\hline Age (years) & & & & 0.564 \\
\hline$<65$ & 78 & 42 & 36 & \\
\hline$\geq 65$ & 77 & 45 & 32 & \\
\hline Gender & & & & 0.401 \\
\hline Male & 83 & 44 & 39 & \\
\hline Female & 72 & 43 & 29 & \\
\hline BMI & & & & 0.647 \\
\hline$<30$ & 83 & 48 & 35 & \\
\hline$\geq 30$ & 72 & 39 & 33 & \\
\hline Clinical stage & & & & $<0.001^{* * *}$ \\
\hline I & 22 & 19 & 3 & \\
\hline$\|$ & 61 & 28 & 33 & \\
\hline III & 67 & 40 & 27 & \\
\hline IV & 5 & 0 & 5 & \\
\hline
\end{tabular}

by the down-regulation in $\mathrm{p}-J \mathrm{NK}$ and $\mathrm{p}$-c-Jun expressions. Conversely, over-expression of MPS-1-enhanced activities of both JNK and c-Jun compared with the negative control in Caco2 cells (Fig. 5b). In consistent, the expressions of p-JNK and p-c-Jun detected by IHC staining in clinical specimens showed generally higher levels in tumors than in the normal tissues (Fig. 5c). In addition, the inhibition effect on JNK/c-Jun signaling of MPS-1 knockdown was partially recovered in response to leptin exposure (Fig. 5a). Further detection showed that the effects of MPS-1 knockdown on proliferation (52.73\% recovery), colony formation (68.31\% recovery), and apoptosis (37.63\% recovery) of RKO cells were significantly compromised by treatment of the JNK activator, anisomycin $(P<0.01$, Fig. $5 \mathrm{~d}-\mathrm{f})$. The results indicated that MPS-1 mediated leptin-induced development of CRC via activation of JNK/c-Jun-signaling pathway.

\section{MPS-1 silencing suppressed tumor formation of CRC in xenografts}

Lastly, to confirm that the in vitro findings are relevant in vivo, RKO cells transfected with shMPS-1 or shCtrl were injected into mice, respectively, for constructing mice xenograft model. As shown in Fig. 6a-c, mice implanted with RKO cells with MPS-1 knockdown exhibited much slower growth and smaller size of tumors $(84.29 \%$ inhibition of volume, $81.42 \%$ inhibition of weight, $P<0.01$ ). In consistent, the tumors formed in shMPS-1 group displayed lower Ki-67 expression than those in shCtrl group (Fig. 6d). In addition, the suppression of activities of JNK and c-Jun

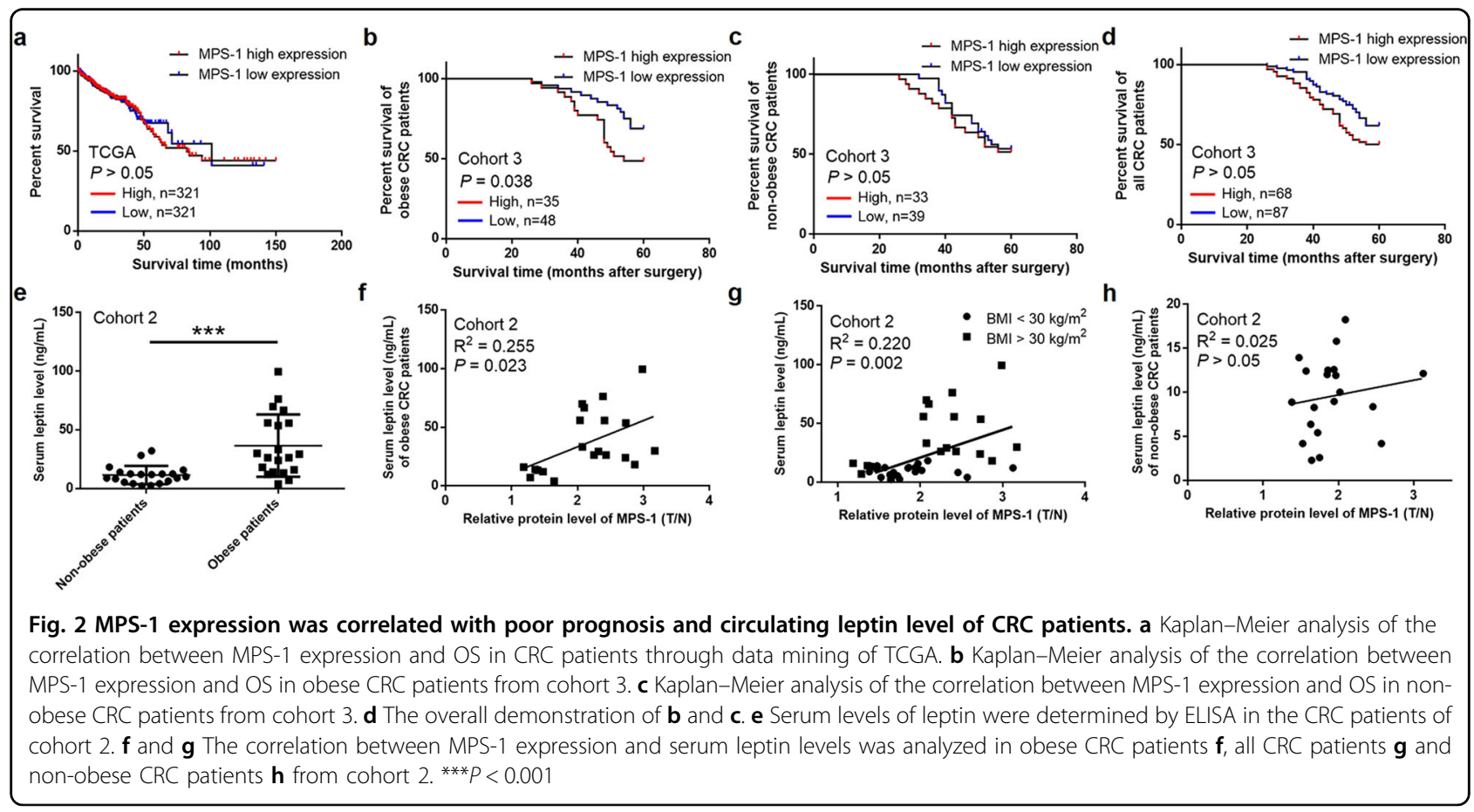




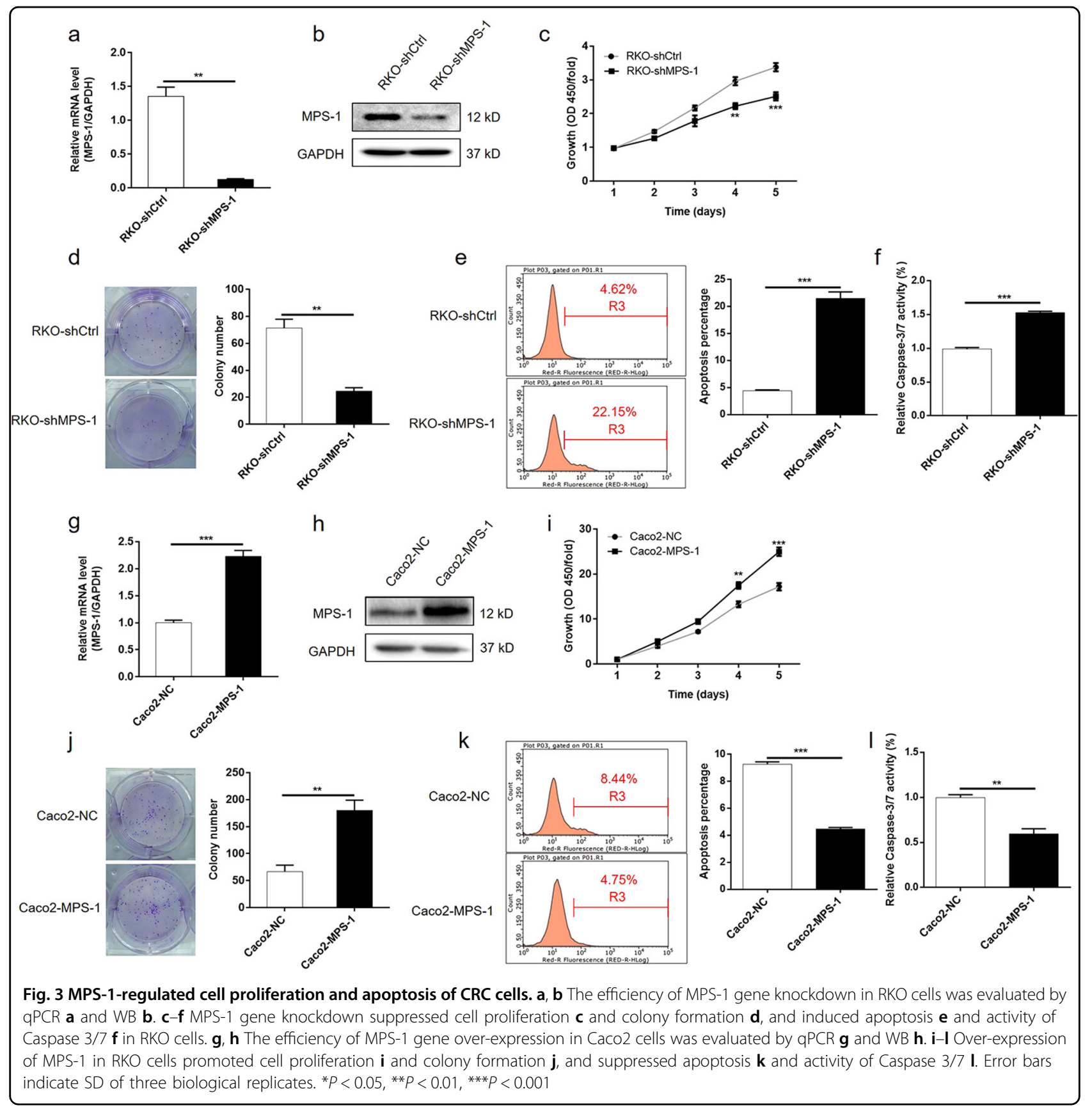

was observed in shMPS-1 group, since expressions of pJNK and p-c-Jun were down-regulated (Fig. 6e). This indicated that MPS-1 knockdown suppressed tumor formation of CRC via suppressing JNK/c-Jun-signaling pathway. Thus, in vivo experiments confirmed that MPS-1 played a crucial role in tumor progression of CRC via activation of JNK/cJun-signaling pathway.

\section{Discussion}

CRC ranks the third most frequently diagnosed cancer worldwide, contributing to a crucial cause of cancer- related death and substantial socioeconomic burden ${ }^{15}$. Although a growing number of therapeutic strategies have been developed over the past decades, the prognosis of CRC is still poor and frustrating ${ }^{16}$. Hence, in-depth investigation into underlying mechanisms is needed to combat the relapse of CRC and improve the prognosis.

In this study, we identified MPS-1 as a candidate promotor in CRC through microarray and gene knockdown experiments. Meanwhile, correlation analysis showed an association between MPS-1 expression and advanced tumor stage, suggesting its association with CRC 


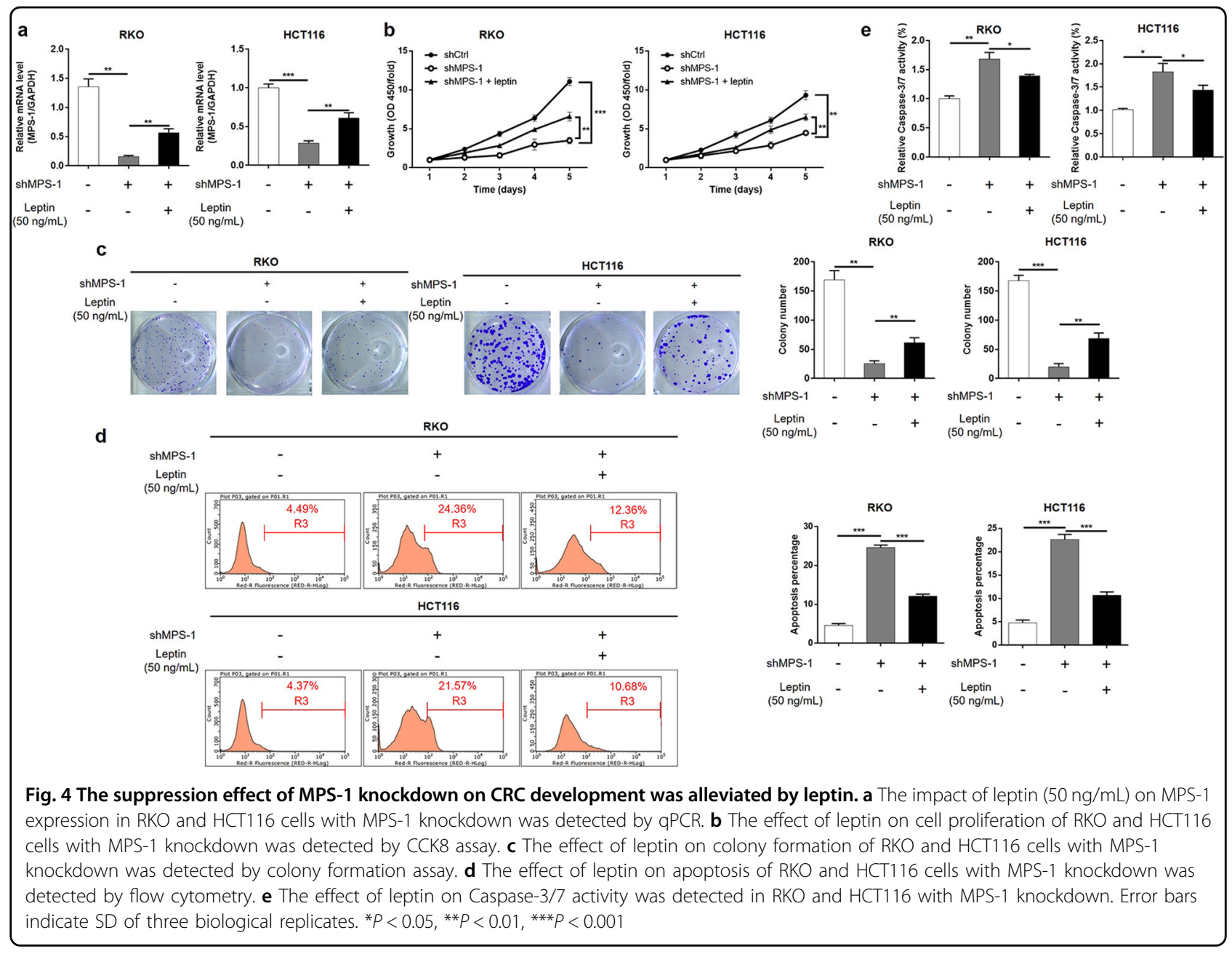

progression and the potential as a prognostic marker. Previous studies showed variable roles of MPS-1 in the different cancer types. For example, while MPS-1 was a tumor suppresser in head and neck squamous cell carcinoma ${ }^{6}$, MPS-1 over-expression was observed in breast cancer $^{7,8}$ and gastric cancer ${ }^{9-11}$. Until now, little was known on the role of MPS- 1 in CRC. The only literature was reported by Ganger et al. illustrating a relationship between MPS-1 over-expression in colonic mucosa crypts and the aggressive behavior of CRC in a patient ${ }^{12}$. However, evidence from cohort study and functional experiment is lacking and warranted. Interestingly, another member of RPS27 family, RPS27a, is highly expressed in CRC and supposed as an early response gene, which might be an indirect support for the implication of MPS-1 in $\mathrm{CRC}^{17}$. The current study exhibited that MPS-1 functions to induce the proliferation and suppress apoptosis in CRC cells in vitro. In vivo experiments demonstrated significant suppression in tumor growth by MPS-1 knockdown. In consistent, Ki-67 expression was decreased in the tumors formed in shMPS-1 group, indicating a lower tumor proliferative fraction in this situation. These results demonstrated that MPS-1 plays a promotion role in CRC development.

Obesity is widespread recognized as a major epigenetic cause for $\mathrm{CRC}^{1}$. Since obesity is a global epidemic and is predicted to continue to increase, it is important to dissect the mechanisms behind to prevent the increasing trend in obesity-associated CRC. Among the regulatory pathways, the complex role of adipokines (such as leptin and adiponectin) secreted by excess adipose tissue is a focus for research $^{1,18}$. In the current study, the up-regulation of MPS-1 expression was observed by microarray screening and IHC verification in clinical specimens. However, data mining of TCGA showed no statistical significance in survival analysis. Intriguingly, in the subgroup analysis according to BMI, MPS-1 was associated with poor prognosis in obese CRC patients, but not in their nonobese counterparts. Subgroup analysis also found a higher fold up-regulation of MPS-1 expression in tumor tissues compared with corresponding normal tissues in the context of obesity. Therefore, we hypothesized an 


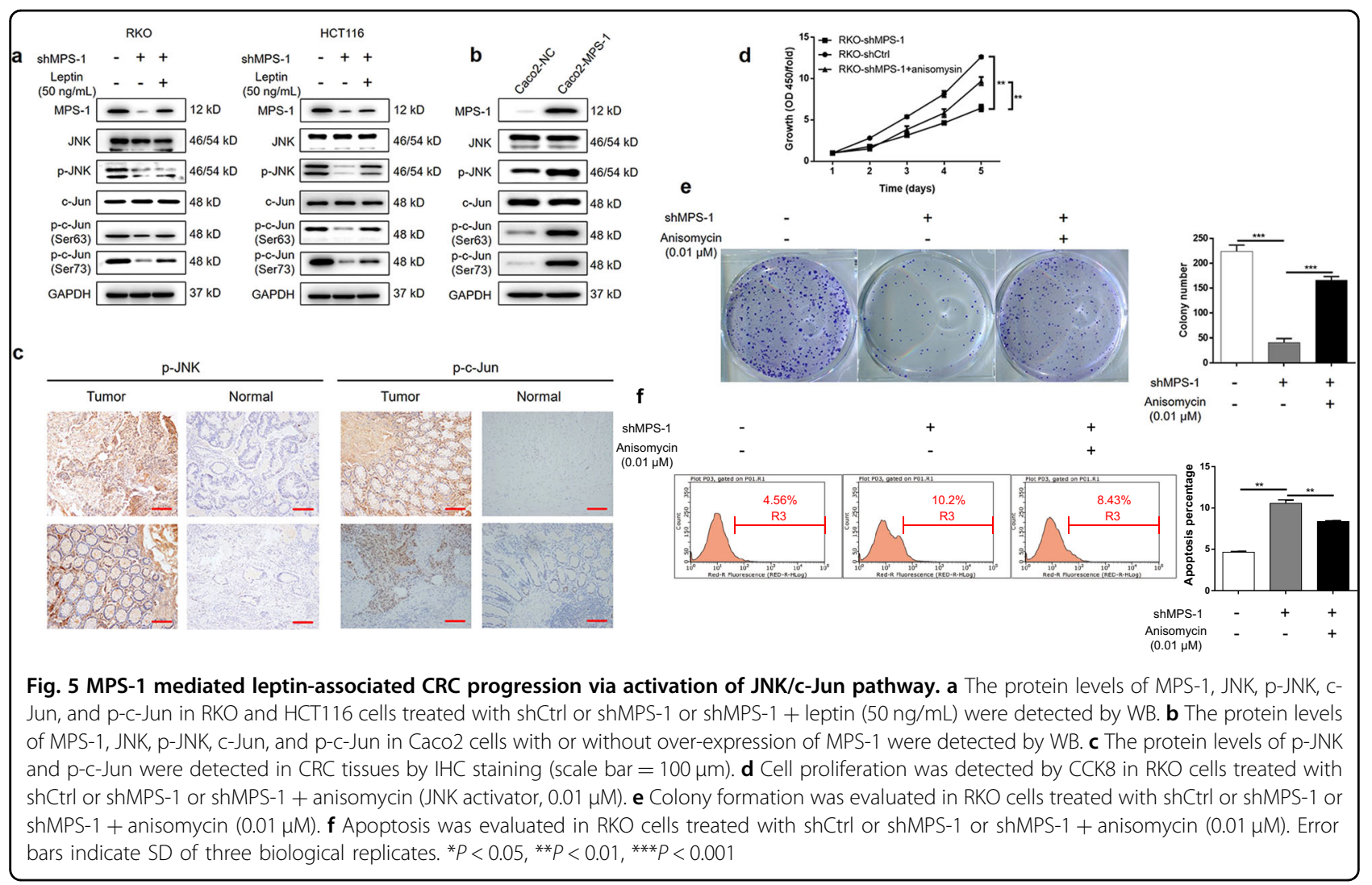

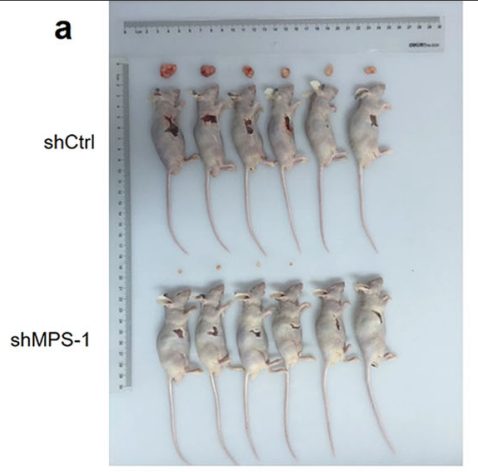

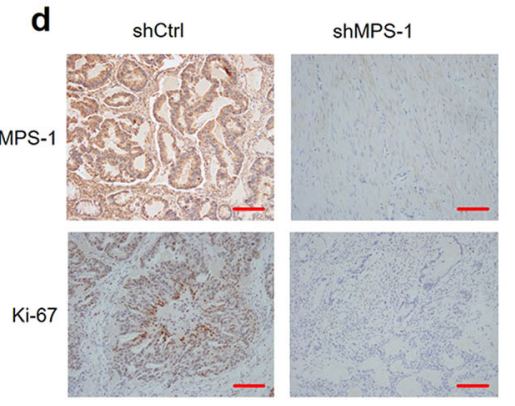

b

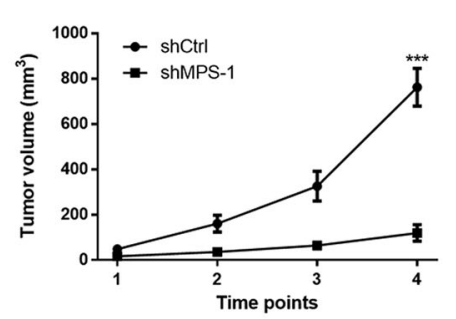

e
C

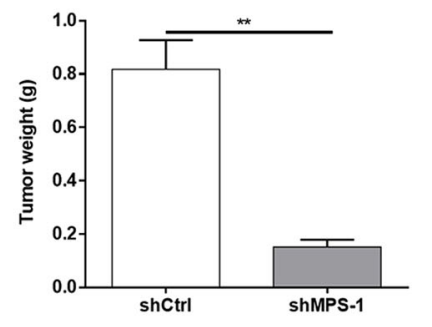

Fig. 6 MPS-1 knockdown suppressed tumor growth in vivo. a The tumor images taken from mice in shCtrl and shMPS-1 groups. $\mathbf{b}$ Tumor volume was measured in shCtrl and shMPS-1 groups at the indicated time points $(6,12,18,24$ days post-injection). c Tumor weights were measured in shCtrl and shMPS-1 groups after sacrificing mice. $\mathbf{d}$ The levels of MPS-1 and Ki-67 were analyzed by IHC staining (scale bar $=100 \mu \mathrm{m}$ ). $\mathbf{f}$ The protein expressions of MPS-1, JNK, p-JNK, c-Jun, and p-c-Jun (Ser63 and Ser73) were detected by WB in the tumors of shCtrl and shMPS-1 groups. Error bars indicate SD of three biological replicates. ${ }^{*} P<0.05,{ }^{* *} P<0.01,{ }^{* *} P<0.001$ 


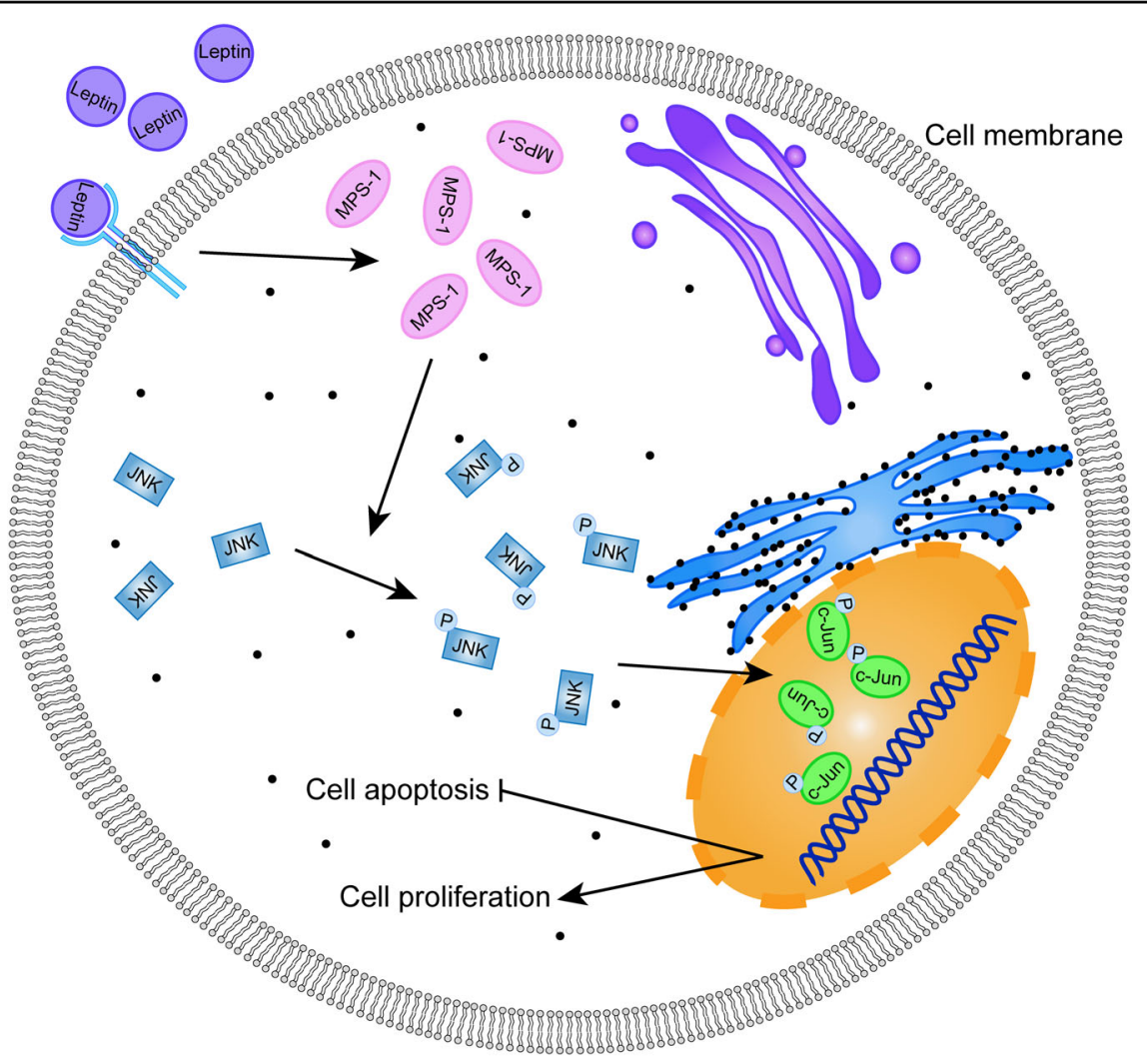

Fig. 7 Schematic diagram of MPS-1 mediated leptin-induced CRC via activation of JNK/C-Jun signaling pathway

association between MPS-1 and obesity-driven CRC development, and focused on this compartment.

MPS-1 is a growth factor-inducible gene, but its upstream regulator is unclear. In this study, we revealed that leptin interferes with the effects of MPS-1 in the progression of CRC. Leptin increases in proportion to the level of obesity ${ }^{3}$, and has profound effects in various cancers $^{14}$. For example, Garofalo et al. reported the upregulation of leptin and its receptor in breast cancer, and identified leptin as a biomarker for tumor progression ${ }^{19}$. It was also demonstrated that leptin promoted the development of lung cancer via JAK/STAT3 pathway ${ }^{13,20}$. Importantly, epidemiological data has established a link between leptin over-expression and high risk of $\mathrm{CRC}^{21}$. Moreover, the promotion function of leptin on growth and migration of CRC has been elucidated ${ }^{22-25}$. In consistent, we found that MPS-1 was correlated with circulating leptin level in CRC patients, especially in obese cases, but not in nonobese cases. In addition, the influence of MPS-1 knockdown on CRC cells was alleviated in response to leptin exposure. Our findings identify MPS-1 as a new player in promoting the development of leptin-mediated CRC.

In this study, we found that MPS-1 silencing inhibited cell growth and induced apoptosis of CRC cells by suppressing JNK/c-Jun pathway, while MPS-1 over-expression displayed the opposite effects by stimulating the signal transduction pathway. Besides, anisomycin, a JNK activator, reversed the inhibition effects of MPS-1 knockdown. These results indicated that MPS-1 is able to stimulate JNK/c-Jun pathway. JNK belongs to the mitogen-activated protein kinase (MAPK) family, and is activated in response to cellular stress $^{26}$. JNK pathway is extensively involved in malignancies including $\mathrm{CRC}^{27,28}$. For example, TASIN-1, an inhibitor of mutant adenomatous polyposis coli (APC) gene, suppresses CRC through cholesterol depletion via JNK pathway ${ }^{29}$. JNK signaling is linked with obesity-driven disorders such as insulin resistance and chronic inflammation ${ }^{30}$. Endo et al. found higher JNK activity in the mice fed with high fat diet (HFD) than in normal diet (ND) group. The effect of JNK on proliferation of colon cells could be suppressed under HFD but not ND, suggesting its role in obesity-associated $\mathrm{CRC}^{31}$. Recent evidences support the involvement of JNK signaling in leptin-related cancers such as ovarian cancer ${ }^{32}$, prostate cancer $^{33}$ and $\mathrm{CRC}^{23}$. However, little is known about the exact mechanism. The current study demonstrated that MPS-1 exerts its action in leptin-associated CRC via JNK/cJun pathway. Our findings further advanced the understanding of leptin-mediated CRC.

There are some limitations which should be taken into account in the interpretation of these results. First, cohort 
1 for microarray analysis has a small sample size. The subsequent analysis in cohort 2 and cohort 3 , however, established a correlation between MPS-1 and leptininduced CRC progression. Second, the mechanism underlying the interaction between MPS-1 and leptin needs further exploration. Third, it is noteworthy that obesity is associated with various adipokines besides leptin. So far, little is known on the regulatory effects of other adipokines on the function of MPS-1 in CRC. In the future, further studies should be conducted for detailed functional assessment of MPS-1 in leptin-mediated CRC.

In conclusion, our findings provide insights of the promoting role of MPS-1 in leptin-induced CRC development via activation of JNK/C-Jun-signaling pathway (Fig. 7). MPS-1 might represent a potent candidate for the treatment and prognostic prediction of CRC patients, especially obese CRC cases.

\section{Acknowledgements}

This work was financially supported by the National Natural Science Foundation of China (No. 81201806) and the Natural Science Foundation of Shanghai Science and Technology Commission (No. 12ZR1442000).

\section{Author contributions}

D.C. and M.Z. designed this study. M.Z. supervised the progress throughout this study. D.C., Y.L., M.Y., S.Q. and J.C. performed all the in vitro and in vivo experiments. M.Y., J.Q., R.C., G.Y., N.Y., Z.C., R.J. and H.C. operated the data analysis in this study. D.C. and Y.L. made this manuscript which was examined and revised by M.Z. All authors have approved the submission of this manuscript.

\section{Conflict of interest}

The authors declare that they have no conflict of interest.

\section{Publisher's note}

Springer Nature remains neutral with regard to jurisdictional claims in published maps and institutional affiliations.

Supplementary Information accompanies this paper at (https://doi.org/ 10.1038/s41419-019-1911-8).

Received: 16 April 2019 Revised: 24 August 2019 Accepted: 26 August 2019 Published online: 10 September 2019

\section{References}

1. Tarasiuk, A., Mosińska, P. \& Fichna, J. The mechanisms linking obesity to colon cancer: an overview. Obes. Res. Clin. Pract. 12, 251-259 (2018).

2. Martinezuseros, J. \& Garciafoncillas, J. Obesity and colorectal cancer: molecular features of adipose tissue. J. Transl. Med. 14, 1-12 (2016).

3. Wasim, M., Awan, F. R., Najam, S. S., Khan, A. R. \& Khan, H. N. Role of leptin deficiency, inefficiency, and leptin receptors in obesity. Biochem. Genet. 54, 1-8 (2016).

4. Song, M. et al. Plasma adiponectin and soluble leptin receptor and risk of colorectal cancer: a prospective study. Cancer Prev. Res. 6, 875-885 (2013).

5. Kenmochi, N. et al. A map of 75 human ribosomal protein genes. Genome Res. 8, 509 (1998).

6. Dai, Y., Pierson, S. E., Dudney, W. C. \& Jr, S. B. Extraribosomal function of metallopanstimulin-1: reducing paxillin in head and neck squamous cell carcinoma and inhibiting tumor growth. Int. J. Cancer 126, 611-619 (2010).
7. Atsuta, Y. et al. Identification of metallopanstimulin-1 as a member of a tumor associated antigen in patients with breast cancer. Cancer Lett. 182, 101-107 (2002).

8. Richardson, A. L. et al. X chromosomal abnormalities in basal-like human breast cancer. Cancer Cell 9, 121-132 (2006).

9. Yang, Z. Y. et al. Metallopanstimulin-1 regulates invasion and migration of gastric cancer cells partially through integrin $\beta 4$. Carcinogenesis 34, 2851 (2013).

10. Wang, Y. et al. In vitro and In vivo evidence of metallopanstimulin-1 in gastric cancer progression and tumorigenicity. Clin. Cancer Res. 12, 4965-4973 (2006).

11. Yang, Z. Y. et al. Knockdown of metallopanstimulin-1 inhibits NF-KB signaling at different levels: the role of apoptosis induction of gastric cancer cells. Int. J. Cancer 130, 2761 (2012).

12. Ganger, D. R., Hamilton, P. D., Fletcher, J. W. \& Fernandez-Pol, J. A. Metallopanstimulin is overexpressed in a patient with colonic carcinoma. Anticancer Res. 17, 1993-1999 (1997).

13. Mullen, M. \& Gonzalez-Perez, R. R. Leptin-induced JAK/STAT signaling and cancer growth. Vaccines 4, 26-42 (2016).

14. Ray, A. \& Cleary, M. P. The potential role of leptin in tumor invasion and metastasis. Cytokine Growth Factor Rev. 38, 80-97 (2017).

15. Bray, F. et al. Global cancer statistics 2018: GLOBOCAN estimates of incidence and mortality worldwide for 36 cancers in 185 countries. CA-Cancer J. Clin. 68, 394-424 (2018).

16. Siegel, R. L. et al. Colorectal cancer statistics, 2017. CA-Cancer J. Clin. 67, 104-117 (2017)

17. Wong, J. M. et al. Ubiquitin-ribosomal protein S27a gene overexpressed in human colorectal carcinoma is an early growth response gene. Cancer Res. $\mathbf{5 3}$, 1916-1920 (1993)

18. Wen, Y. A. et al. Adipocytes activate mitochondrial fatty acid oxidation and autophagy to promote tumor growth in colon cancer. Cell Death Dis. 8, e2593 (2017).

19. Garofalo, C. et al. Increased expression of leptin and the leptin receptor as a marker of breast cancer progression: possible role of obesity-related stimuli. Clin. Cancer Res. 12, 1447-1453 (2006).

20. Zheng, X. et al. Downregulation of leptin inhibits growth and induces apoptosis of lung cancer cells via the Notch and JAK/STAT3 signaling pathways. Biol. Open 5, 794-800 (2016).

21. Mariusz, K., Mariola, S., Luiza, K. K., Eva, S. \& Stanislaw, S. Overexpression of the obesity hormone leptin in human colorectal cancer. J. Clin. Pathol. 60 902-906 (2007).

22. Mohammad, A. et al. Leptin receptor-related immune response in colorectal tumors: the role of colonocytes and interleukin-8. Cancer Res. 68, 9423-9432 (2008).

23. Ogunwobi, O. O. \& Beales, I. L. P. The anti-apoptotic and growth stimulatory actions of leptin in human colon cancer cells involves activation of JNK mitogen activated protein kinase, JAK2 and PI3 kinase/Akt. Int. J. Colorectal Dis. 22, 401-409 (2007)

24. Drew, J. E. Molecular mechanisms linking adipokines to obesity-related colon cancer: focus on leptin. Proc. Nutr. Soc. 71, 175-180 (2012).

25. Zhou, W., Tian, Y., Gong, H., Guo, S. \& Luo, C. Oncogenic role and therapeutic target of leptin signaling in colorectal cancer. Expert Opin. Ther. Tar. 18, 961-971 (2014)

26. Mauricio, B., Chiou, V. L., Jung-Min, L. \& Kohn, E. C. The MAPK pathway across different malignancies: a new perspective. Cancer-Am. Cancer Soc. 120, 3446-3456 (2015)

27. Wang, B. et al. LASP2 suppresses colorectal cancer progression through JNK p38 MAPK pathway meditated epithelial-mesenchymal transition. Cell Commun. Signal. 15, 21 (2017).

28. Liao, Y. et al. Nuclear receptor binding protein 1 correlates with better prognosis and induces caspase-dependent intrinsic apoptosis through the JNK signalling pathway in colorectal cancer. Cell Death Dis. 9, 436-452 (2018).

29. Zhang, L., Kim, S. B., Luitel, K. \& Shay, J. W. Cholesterol depletion by TASIN-1 induces apoptotic cell death through the ER stress/ROS/JNK signaling in colon cancer cells. Mol. Cancer Ther. 17, 943-951 (2018).

30. Han, M. S. et al. JNK expression by macrophages promotes obesity-induced insulin resistance and inflammation. Science 339, 218-222 (2013).

31. Endo, H. et al. Involvement of JNK pathway in the promotion of the early stage of colorectal carcinogenesis under high-fat dietary conditions. Gut 58, 1637-1643 (2009). 
32. Ghasemi, A., Hashemy, S. I., Aghaei, M. \& Panjehpour, M. Leptin induces matrix metalloproteinase 7 expression to promote ovarian cancer cell invasion by activating ERK and JNK pathways. J. Cell. Biochem. 119, 2333-2344 (2018).
33. Miyazaki, T., Bub, J. D., Iwamoto, Y. \& c-Jun, N. H. (2)-terminal kinase mediates leptin-stimulated androgen-independent prostate cancer cell proliferation via signal transducer and activator of transcription 3 and Akt. Biochim. Biophys. Acta 1782, 593-604 (2008). 